Louisiana State University

LSU Digital Commons

Faculty Publications

Department of Physics \& Astronomy

$1-1-2020$

\title{
New Ab Initio Approach to Nuclear Reactions Based on the Symmetry-Adapted No-Core Shell Model
}

\author{
Alexis Mercenne \\ Louisiana State University \\ Kristina D. Launey \\ Louisiana State University \\ Jutta E. Escher \\ Lawrence Livermore National Laboratory \\ Tomas Dytrych \\ Louisiana State University \\ Jerry P. Draayer \\ Louisiana State University
}

Follow this and additional works at: https://digitalcommons.Isu.edu/physics_astronomy_pubs

\section{Recommended Citation}

Mercenne, A., Launey, K., Escher, J., Dytrych, T., \& Draayer, J. (2020). New Ab Initio Approach to Nuclear Reactions Based on the Symmetry-Adapted No-Core Shell Model. Springer Proceedings in Physics, 238, 253-257. https://doi.org/10.1007/978-3-030-32357-8_44

This Conference Proceeding is brought to you for free and open access by the Department of Physics \& Astronomy at LSU Digital Commons. It has been accepted for inclusion in Faculty Publications by an authorized administrator of LSU Digital Commons. For more information, please contact ir@lsu.edu. 
New Ab Initio Approach to Nuclear Reactions Based on the Symmetry-Adapted No-Core Shell Model

\author{
A. Mercenne, K. D. Launey, J. E. Escher, T. \\ Dytrych, J. P. Draayer
}

December 5, 2018

XXII International Conference on Few-Body Problems in Physics

Caen, France

July 9, 2018 through July 13, 2018 
This document was prepared as an account of work sponsored by an agency of the United States government. Neither the United States government nor Lawrence Livermore National Security, LLC, nor any of their employees makes any warranty, expressed or implied, or assumes any legal liability or responsibility for the accuracy, completeness, or usefulness of any information, apparatus, product, or process disclosed, or represents that its use would not infringe privately owned rights. Reference herein to any specific commercial product, process, or service by trade name, trademark, manufacturer, or otherwise does not necessarily constitute or imply its endorsement, recommendation, or favoring by the United States government or Lawrence Livermore National Security, LLC. The views and opinions of authors expressed herein do not necessarily state or reflect those of the United States government or Lawrence Livermore National Security, LLC, and shall not be used for advertising or product endorsement purposes. 


\title{
New ab initio approach to nuclear reactions based on the symmetry-adapted no-core shell model
}

\author{
Alexis Mercenne ${ }^{1}$, Kristina D. Launey ${ }^{1}$, Jutta E. Escher ${ }^{2}$, Tomas Dytrych ${ }^{1,3}$, \\ and Jerry P. Draayer ${ }^{1}$ \\ 1 Department of Physics and Astronomy, Louisiana State University, Baton Rouge, \\ LA 70803, USA, \\ amercenne1@lsu.edu \\ 2 Lawrence Livermore National Laboratory, Livermore, CA, 94550, USA \\ 3 Nuclear Physics Institute, Academy of Sciences of the Czech Republic, 25068 Rež, \\ Czech Republic
}

\begin{abstract}
We present the current development of a new ab initio approach for nuclear reactions that takes advantage of $\mathrm{SU}(3)$ symmetry and its relevant dynamics combined with the resonating group method. In this model, the structure of the clusters is based on the ab initio symmetry-adapted no-core shell model, which enables the description of spatially enhanced nuclear configurations. We will present the formalism that involves the expression of the norm kernels in the SU(3) symmetryadapted basis, in addition to first results for the $\mathrm{p}-\alpha, \mathrm{p}-{ }^{16} \mathrm{O}$ and $\mathrm{p}-{ }^{20} \mathrm{Ne}$ scattering reactions.
\end{abstract}

Keywords: Nuclear structure, nuclear reactions, group theory, astrophysics

Introduction: The development of new experimental facilities has highlighted the need for new microscopic nuclear reaction models. In addition, recent progresses in ab initio nuclear theory using realistic, QCD inspired, interactions as well as many developments in high performance computing (HPC) have given the necessary tools to theoretical approaches such as the no-core shell model to provide an $a b$ initio description of the structure of light nuclei [1,2]. Its recent implementation within the resonating group method (RGM) [3] has allowed a microscopic study of nuclear reactions $[4,5]$, pursuing the long-lasting goal to unify the nuclear structure and reactions. However, even with the development of more advanced HPC techniques, the nuclear structure and reactions for certain mass region remain out of reach for $a b$ initio approaches mainly due to the the size of the configuration space. Recently, the symmetry-adapted no-core shell model (SA-NCSM) $[6,7]$ which considers a more physically relevant basis, has proven its efficacy and has been successfully applied to the description of nuclear structure for nuclei up to medium mass. Motivated by the need for calculated nuclear cross sections in experimental research and astrophysics studies, and following the spirit of the NCSM/RGM, we combine the SA-NCSM with 
the RGM, with the view toward providing a complete description of structure and reactions for binary reactions in which the projectile is a nucleon.

Unified Ab Initio Approach For Medium-mass Nuclei: In the RGM, the wave function is expanded within a clusters basis:

$$
\left|\Psi^{J^{\pi} T}\right\rangle=\sum_{\nu} \int_{r} d r r^{2} \frac{g_{\nu}^{J^{\pi} T}(r)}{r} \hat{\mathcal{A}}\left|\Phi_{\nu r}^{J^{\pi} T}\right\rangle,
$$

where the index $\nu$ gathers all quantum numbers defining channels and partitions: $\nu=\left\{(A-a) \alpha_{1} I_{1} T_{1} ; a \alpha_{2} I_{2} T_{2} ; \ell s\right\}$, and the cluster states are defined as $\left|\Phi_{\nu r}^{J^{\pi} T}\right\rangle=\left[\left(\left|(A-a) \alpha_{1} I_{1} T_{1}\right\rangle \otimes\left|a \alpha_{2} I_{2} T_{2}\right\rangle\right)^{(s T)} \times Y_{\ell}\left(\hat{r}_{A-a, a}\right)\right]^{\left(J^{\pi} T\right)} \frac{\delta\left(r-r_{A-a, a}\right)}{r r_{A-a, a}}$. The wave functions $g_{\nu}^{J^{\pi} T}(r)$ in Eq.(1) are the quantities to be determined. They describe the relative motion between the target and the projectile for all channels $\nu$, and the cross section can be extracted from their asymptotic behavior. The determination of $g_{\nu}^{J^{\pi} T}(r)$ is achieved by solving the Schrödinger equation:

$$
\sum_{\nu} \int d r r^{2}\left[H_{\nu^{\prime} \nu}^{J^{\pi} T}\left(r, r^{\prime}\right)-E N_{\nu^{\prime} \nu}^{J^{\pi} T}\left(r^{\prime}, r\right)\right] \frac{g_{\nu}^{J^{\pi} T}(r)}{r}=0 .
$$

Here, the Hamiltonian $H_{\nu^{\prime} \nu}^{J^{\pi} T} T\left(r^{\prime}, r\right)$ and norm ${N_{\nu^{\prime} \nu}^{J^{\pi}} T}^{*}\left(r^{\prime}, r\right)$ kernels are expressed as: $\left\langle\Phi_{\nu^{\prime} r^{\prime}}^{J^{\pi} T}|\hat{\mathcal{A}} \hat{O} \hat{\mathcal{A}}| \Phi_{\nu r}^{J^{\pi} T}\right\rangle$, where $\hat{\mathcal{A}}$ is the antisymmetrizer ensuring the Pauli exclusion principle, and they are computed using the wave functions of the clusters. Once the kernels are computed within a given basis, Eq.(2) can then be solved using an $R$-matrix approach.

An $a b$ initio application of this approach is the NCSM/RGM which uses $a b$ initio NCSM wave functions generated using realistic interactions in order to compute the kernels. The NCSM/RGM has then been succesfully applied to the description of several nuclear reactions involving light nuclei. However the method becomes numerically challenging for heavier systems due to the size and complexity of the configuration space. In addition, the inversion of the norm kernel as well as the treatment of the center-of-mass excitations become challenging tasks when the number of channels increases. In this context, the SA-NCSM combined with the RGM holds promise to obtain a unified ab initio description of structure and reaction for intermediate- up to medium-mass nuclei.

In the SA-NCSM, the microscopic many-body basis (Slater determinants) is based on the spherical harmonic oscillator single particle basis. In our case, we consider a basis made of the irreductible representations according to the group chain: $\mathrm{SU}(3)_{(\lambda \mu)} \underset{\kappa}{\supset} \mathrm{SO}(3)_{L} \supset \mathrm{SO}(2)_{M_{L}}$. Consequently, for any given total spin and its projection $J M$, the wave function of a nucleus will be described within a basis $\left\{\left|\alpha_{i}\left(\lambda_{i} \mu_{i}\right) \kappa_{i}\left(L_{i} S_{i}\right) J_{i} M_{i}\right\rangle\right\}$ with each components weighted by a coefficient $C_{i}$, and where $\alpha_{i}$ gathers additional quantum numbers needed to enumerate the complete shell model space.

In the symmetry-adapted RGM (SA-RGM), the channels are defined by coupling each components of the SA-NCSM wave functions between the projectile 
and the target. Consequently, the channels with good $\mathrm{SU}(3)$, spin and isospin quantum numbers are given in the case of one nucleon projectile as:

$$
\left|\Phi_{\gamma n}^{\rho(\lambda \mu) \kappa(L S) J M T M_{T}}\right\rangle=\left\{\left|\alpha_{1}\left(\lambda_{1} \mu_{1}\right) S_{1} T_{1}\right\rangle \otimes\left|(n 0) \frac{1}{2} \frac{1}{2}\right\rangle\right\}^{\rho(\lambda \mu) \kappa(L S) J M T M_{T}},
$$

where the index: $\gamma \equiv\left\{(A-a) \alpha_{1}\left(\lambda_{1} \mu_{1}\right) S_{1} T_{1} ; a \frac{1}{2} \frac{1}{2}\right\}$ label our channel basis, $(n 0)$ represents the $\mathrm{SU}(3)$ relative motion of the projectile, and $\left(\frac{1}{2} \frac{1}{2}\right)$ its spin and isospin respectively. In this basis, the exchange matrix, which ensures the antisymmetrization in the kernels, has the following form (in conventional notations $[8]):$

$$
\begin{aligned}
& \left\langle\Phi_{\gamma^{\prime} n^{\prime}}^{\rho^{\prime}\left(\mu^{\prime} \mu^{\prime}\right) \kappa^{\prime}\left(L^{\prime} S^{\prime}\right) J M T M_{T}}\left|\hat{P}_{A, A-1}\right| \Phi_{\gamma n}^{\left.\rho(\lambda \mu) \kappa(L S) J M T M_{T}\right\rangle}\right. \\
& =\frac{1}{A-1} \delta_{\rho \rho^{\prime}} \delta_{(\lambda \mu)\left(\lambda^{\prime} \mu^{\prime}\right)} \delta_{\kappa \kappa^{\prime}} \delta_{L L^{\prime}} \delta_{S S^{\prime}} \sum_{\tau \rho_{o}\left(\lambda_{o} \mu_{o}\right)} \Pi_{\tau S_{o} S_{1}^{\prime} T_{1}^{\prime}}(-1)^{n+n^{\prime}-\left(\lambda_{o}+\mu_{o}\right)} \\
& \times(-1)^{T_{1}+\frac{1}{2}+T^{\prime}}(-1)^{S_{1}+\frac{1}{2}+S^{\prime}}\left\{\begin{array}{ccc}
S_{1} & S_{o} & S_{1}^{\prime} \\
\frac{1}{2} & S & \frac{1}{2}
\end{array}\right\}\left\{\begin{array}{c}
T_{1} \tau T_{1}^{\prime} \\
\frac{1}{2} T
\end{array}\right\} \\
& \times \sqrt{\frac{\operatorname{dim}\left(\lambda_{o} \mu_{o}\right)}{\operatorname{dim}(n 0)}} \mathrm{U}\left[\left(\lambda_{1} \mu_{1}\right)\left(\lambda_{o} \mu_{o}\right)\left(\lambda^{\prime} \mu^{\prime}\right)\left(n^{\prime} 0\right) ;\left(\lambda_{1}^{\prime} \mu_{1}^{\prime}\right) \bar{\rho} \rho^{\prime}(n 0) \rho_{o} \rho^{\prime \prime}\right] \\
& \times\left\langle\alpha_{1}^{\prime}\left(\lambda_{1}^{\prime} \mu_{1}^{\prime}\right) S_{1}^{\prime} T_{1}^{\prime} \mid\left\|\left\{a_{(n 0) \frac{1}{2} \frac{1}{2}}^{\dagger} \otimes \tilde{a}_{\left(0 \tilde{n}^{\prime}\right)} \frac{1}{2} \frac{1}{2}\right\}^{\rho_{o}\left(\lambda_{o} \mu_{o}\right) S_{o} \tau}\right\| \alpha_{1}\left(\lambda_{1} \mu_{1}\right) S_{1} T_{1}\right\rangle_{\bar{\rho}} .
\end{aligned}
$$

An important advantage here is that the exchange matrix is diagonal within this $\mathrm{SU}(3)$ basis, and this allows one to overcome numerical inversion of the norm. So with such an approach, the dependence on angular momentum is defered to the very last step in the calculations, and in turn, facilitates quick calculations [9]. Then, only at the end we transform back to the partial waves expansion, i.e $\left|\Phi_{\gamma n}^{\rho(\lambda \mu) K(L S) J M T M_{T}}\right\rangle \rightarrow\left|\Phi_{\nu n}^{J^{\pi} T}\right\rangle$ using the coefficients $C_{i}$, and calculate the norm $N_{\nu^{\prime} \nu}^{J^{\pi} T}\left(r^{\prime}, r\right)$ using the conventional formula [4].

In order to demonstrate the efficacy of the approach, we present a benchmark calculation for $\mathrm{p}-{ }^{4} \mathrm{He}$. We compare the exchange part of the norm (see in Ref.[4]) using the two NCSM/RGM and SA-RGM approaches Fig.(1a). The SA-RGM result has been obtained using a ${ }^{4} \mathrm{He}$ wave function truncated to only several $\mathrm{SU}(3)$ shapes. To illustrate the potential of applying this approach to heavier systems, we present calculations of this exchange part for heavier system in Fig.(1b), for $\mathrm{p}^{-16} \mathrm{O}$ and $\mathrm{p}^{20} \mathrm{Ne}$.

Hence those results show that the use of a physically relevant basis through the SA-RGM is a promising approach, where truncated target wave functions can be implemented to reach heavier system for nuclear reactions So far, the spurious center-of-mass motion between the clusters has not been removed, but it is expected to be negligible for reactions involving one nucleon plus an $A>16$ target. This work presents the method to implement the RGM within an $\mathrm{SU}(3)$ basis, more specifically how to take advantage of the $\mathrm{SU}(3)$ basis to calculate 
Alexis Mercenne et al.
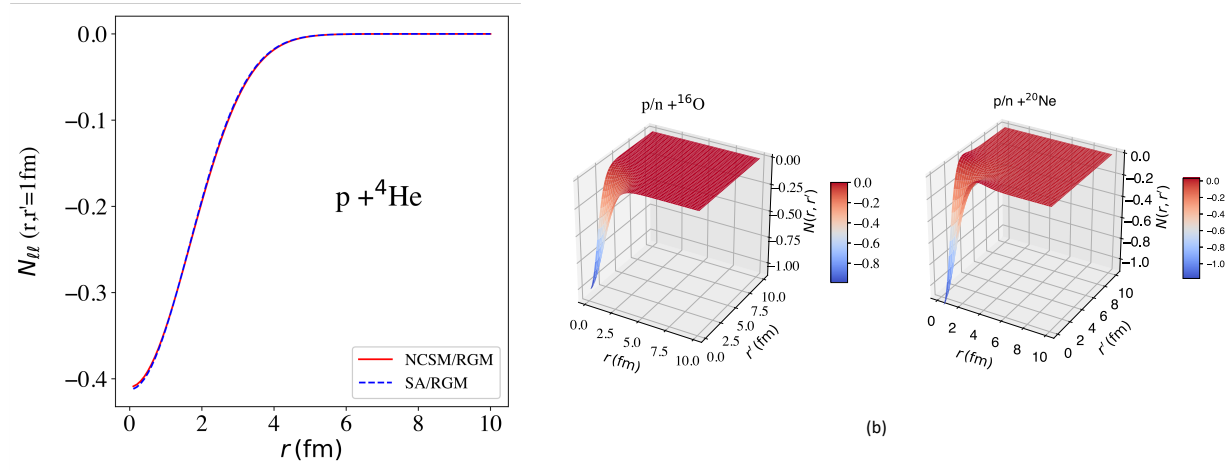

(b)

(a)

Fig. 1. (a) Exchange part of the norm kernel for $\mathrm{p}-{ }^{4} \mathrm{He}$. The target wave function is calculated in a $N_{\max }=4$ model space, and is truncated in the SA-RGM calculation by selecting only components greater than $1 \%$. The calculation of ${ }^{4} \mathrm{He}$ was performed using the chiral $\mathrm{N}_{2} \mathrm{LO}_{\text {opt }} \mathrm{NN}$ interaction. (b) Exchange part of the norm calculated for two heavier system using SA-RGM. Target wave function has been generated using the chiral $\mathrm{N}_{2} \mathrm{LO}_{\text {sat }} \mathrm{NN}$ in 10 shells $(\hbar \Omega=16 \mathrm{MeV})$ for ${ }^{16} \mathrm{O}$ and the chiral $\mathrm{N} 2 \mathrm{LO}_{\text {opt }}$ $\mathrm{NN}$ in 13 shells $(\hbar \Omega=15 \mathrm{MeV})$ for ${ }^{20} \mathrm{Ne}$, with selected $\mathrm{SU}(3)$ configurations that have a contribution greater than $2 \%$. In all calculations, the spurious center-of-mass motion has been removed from the ab initio wave functions, but not from the cluster system.

the norm kernel. The same procedure will be used for the Hamiltonian kernel.

This work was supported by the U.S. National Science Foundation (OIA1738287, ACI -1713690), the Czech Science Foundation (16-16772S) and under the auspices of the U.S. Department of Energy by Lawrence Livermore National Laboratory under Contract DE-AC52- 07NA27344, with support from LDRD project 19-ERD-017. In addition, this work benefitted from computing resources provided by LSU (www.hpc.1su.edu) and Blue Waters.

\section{References}

1. P. Navrátil, J. P. Vary and B. R. Barrett, Phys. Rev. Lett. 84, 5728 (2000)

2. B. R. Barrett, P. Navrátil and J. P. Vary, Prog. Part. Nucl. Phys. 69, 131 (2013)

3. Y. C. Tang, M. LeMere, and D. R. Thompson, Phys. Rep. 47, 167 (1978)

4. S. Quaglioni and P. Navrátil, Phys. Rev. C 79, 044606 (2009)

5. S. Baroni, P. Navrátil and S. Quaglioni, Phys. Rev. Lett. 110, 022505 (2013)

6. T. Dytrych et al., Phys. Rev. Lett. 111, 252501 (2013)

7. K. D. Launey, T. Dytrych and J. P. Draayer, Prog. Part. Nucl. Phys. 89, 101 (2016)

8. J. P. Draayer and Y. Akiyama, J. Math. Phys. 14, 12 (1973)

9. K. T. Hecht, Nucl. Phys. A283, 223 (1977) 